\title{
The Toronto Hospital Alertness Test scale: relationship to daytime sleepiness, fatigue, and symptoms of depression and anxiety
}

This article was published in the following Dove Press journal:

Nature and Science of Sleep

19 January 2016

Number of times this article has been viewed

\author{
Azmeh Shahid ${ }^{1-5}$ \\ Sharon A Chung ${ }^{1,2,5}$ \\ Lance Maresky' \\ Affan Danish' \\ Arina Bingeliene $1,4,5$ \\ Jianhua Shen' \\ Colin M Shapiro ${ }^{1-5}$ \\ 'Sleep Research Laboratory, \\ Department of Psychiatry, \\ University Health Network, \\ University of Toronto, ${ }^{2}$ Youthdale \\ Treatment Centres, ${ }^{3}$ Youthdale \\ Child and Adolescent Sleep Centre, \\ ${ }^{4}$ Department of Psychiatry, University \\ of Toronto, ${ }^{5}$ Department of Psychiatry, \\ Toronto Western Hospital, University \\ Health Network, Toronto, ON, Canada
}

Correspondence: Sharon A Chung Sleep Research Laboratory, Department of Psychiatry, Toronto Western Hospital, University Health Network, 399 Bathurst Street, 7 Main - 429, Toronto,

ON M5T 2S8, Canada

Tel +l 4166035275

Fax + I 4166035292

Email sachung@uhnresearch.ca
Objective: The Toronto Hospital Alertness Test (THAT) scale was designed to measure alertness, defined as the capacity of the mind to respond appropriately to external and internal stimuli. The present study's aim is to determine normative values of alertness on the THAT and to explore the relationship among excessive daytime sleepiness, fatigue, depressive symptoms, and alertness.

Methods: Normative data were collected from 60 healthy males and females. To explore the relationship among alertness, daytime sleepiness, fatigue, depression, and anxiety, data were collected from charts of sleep clinic patients. All study subjects completed measures for fatigue, sleepiness, depressive symptoms, and anxiety.

Results: The average score on the THAT was 34.9 \pm 7.2 (range 22-50) for the control group. The cutoff score for the THAT, indicative of clinically significant reduced alertness, was determined to be $\leq 20.5$ (mean $-2 \mathrm{SD}$ ). THAT alertness scores were found to be modestly, significantly, and negatively correlated with fatigue levels $(r=-0.39, P<0.001)$, depressive symptoms ( $r=-0.53, P<0.001)$, and anxiety symptoms ( $r=-0.41, P<0.001)$. No correlations were found between alertness levels and daytime sleepiness. Regression analyses revealed a significant model $\left(F=19.9, P<0.001\right.$, adjusted $\left.R^{2}=0.35\right)$ with depressive symptoms $(P<0.001)$ and fatigue $(P=0.006)$ emerging as the only significant predictors of scores on the THAT.

Conclusion: The findings of this study support that sleepiness is not the same as poor alertness. Depressive symptoms and fatigue, but not sleepiness, were found to have a strong and significant impact on levels of alertness. This is the first study to link poor alertness to depressive symptoms.

Keywords: alertness, sleepiness, fatigue, depression, anxiety

\section{Introduction}

Alertness is defined as the capacity of the mind to respond appropriately to external and internal stimuli. ${ }^{1}$ Alertness encompasses facets of attention, vigilance, and cognitive performance; individuals who are alert are better able to perceive and react appropriately to varying situations. In essence, excessive sleepiness is defined as a pathologically increased propensity to fall asleep; the operational definition of excessive sleepiness that was provided in a large epidemiological study includes concepts of ease of falling asleep, periods of onset of uncontrollable sleep, falling asleep during what would normally be a period of wakefulness and an increased need to take naps. ${ }^{2}$ Our previous work has proposed that daytime sleepiness and alertness are not opposite states of the same phenomena, but are distinct manifestations of different neural pathways and neurochemical mechanisms. ${ }^{1}$ 
A recently developed measure of alertness, the Toronto Hospital Alertness Test (THAT) scale, has been shown to be valid and have high re-test reliability in the determination of trait alertness levels. ${ }^{1}$ This latter study also provides information on the development of the THAT. In a small study ${ }^{3}$ of 12 healthy volunteers (aged $39 \pm 7$ years), a mean score of $38 \pm 7$ was obtained using the THAT. A larger study of 1,010 normal subjects who were free from obstructive sleep apnea, depression, narcolepsy, multiple sclerosis, shift work, or daytime sleepiness found a mean THAT score of 36.9 $\pm 8.2 .{ }^{4}$ The findings of this latter larger study is promising, but the study did not explore any relationship the THAT scale may have to depression and fatigue. Also, this latter study was also not specifically designed to define a normal range and cutoff for the THAT. As such, there is yet no normal range and cutoff for the THAT has been defined; a reliable normal range for the THAT will require better designed studies.

Our previous study has suggested that no correlation exists between the THAT and polysomnographic (PSG) measure of sleepiness (as measured using the Multiple Wakefulness Test, MWT). ${ }^{1}$ The MWT is a measure best suited to assess one's ability to stay awake. The Maintenance of Wakefulness Test is a PSG measurement of the ability to resist any sleep propensity under soporific conditions and is likely to be yet another measure of the collection of states (eg, sleepiness, alertness, and fatigue). However, Bonnet and Arand $^{5}$ have proposed that the Multiple Sleep Latency Test (MSLT) is a better measure of sleepiness. The MSLT is a measure of sleep propensity, whereas the MWT is a measure of ability to remain awake. ${ }^{6}$

We have previously shown ${ }^{1}$ a modest but significant correlation between alertness (as measured using the THAT) and symptoms of depression (as evaluated using the Centre for Epidemiological Studies in Depression [CESD] screening instrument ${ }^{7}$ ). This finding has resulted in the hypothesis that mood may be a key influence on alertness. Symptoms of anxiety were not examined in the aforementioned study. As such, the aforementioned hypothesis warrants exploration using measures of both anxiety and depressive symptoms.

A further key quandary is the question of what association, if any, exists between cognitive fatigue and alertness. Fatigue is a ubiquitous complaint in mood disorders ${ }^{8,9}$ and in those with sleep pathology. ${ }^{10}$ Fatigue levels have been observed to significantly impact the ability to maintain sustained attention in several patient populations. ${ }^{11,12}$ Yet, the link between fatigue and cognitive performance remains uncertain; some studies found no relationship ${ }^{13,14}$ while others report a negative association. ${ }^{15,16}$
The aim of the present study is to further explore the relationship among alertness, sleepiness mood, and fatigue.

\section{Methods}

Ethics approval for this study was obtained from the University Health Network Research Ethics Board. As this was a retrospective clinical chart review study no informed consent from patients was required as there was no direct contact with patients. To obtain normative values for the THAT, data were collected from 60 healthy males and females ranging in age from 17 to 74 years. To explore the relationship among alertness, sleepiness, fatigue, and symptoms consistent with depression and anxiety, data were also collected from patients who were referred to our clinic for investigation of a sleep disorder and who subsequently underwent overnight PSG testing. Clinical charts were consecutively selected for inclusion in the study - a total of 458 charts were reviewed, but 194 were excluded due to missing data on one or more of the major outcome variables. Patients' charts contained responses to a battery of self-report scales that measured: alertness - THAT; ${ }^{1}$ depressive symptoms - CES-D; ${ }^{7}$ anxiety symptoms - Zung Self-Rating Anxiety Scale (SAS); ${ }^{17}$ daytime sleepiness - Epworth Sleepiness Scale (ESS); ${ }^{18}$ fatigue - Fatigue Severity Scale (FSS). ${ }^{19}$

Results from daytime PSG sleep latency testing (as measured using the MSLT) $)^{20}$ were available for 210 patients. The questionnaires in the charts had been completed on the day of the consultation with the sleep specialist and the PSG testing took place 2-4 weeks later.

The following cutoffs were used for this study: Scores $\geq 16$ is indicative of depression based on the CES-D $;{ }^{7} \leq 44$ represents the normal range of the $\mathrm{SAS} ;{ }^{17} \geq 8$ signifies clinical sleepiness for a sleep clinic population based on the ESS; ${ }^{21}$ and, for the FSS, the normal average healthy adult score is $2.3 \pm 0.7,{ }^{19}$ with a cutoff $>3.7$ (representing the mean $+2 \mathrm{SD}$ ) indicative of pathological fatigue.

\section{Statistical analyses}

Statistical testing was conducted using the SPSS statistical program (version 19, IBM Corporation, Armonk, NY, USA). Differences in means were obtained using the Student's $t$-test. Relationships between variables were explored using Pearson's Correlation and Regression Analyses were performed to determine the degree to which mood (symptoms of depression based on the CES-D and anxiety based on the SAS), daytime sleepiness, fatigue, age, and sex contribute to levels of alertness. The level of statistical significance was set at a more stringent level of $P<0.01$ to avoid Type 1 error due to multiple statistical testing of the dataset. Effect size was 
determined using Cohen's $d^{22}$ which provides the standardized mean effect and expresses the mean difference between two groups in SD units: effect sizes between 0.5 and 0.8 are moderate and above 0.8 are considered large..$^{22}$

\section{Results}

\section{Normative values: control subjects}

Possible scores on the THAT range from 0 to 50. Normative values of the THAT were collected from 30 males and 30 females ranging in age from 17 to 74 years (average $39.1 \pm 14.3$ years). The average score on the THAT was $34.9 \pm 7.2$ (range 22-50). Based on the formula of two times the SD subtracted from the mean, the cutoff of the THAT, indicative of clinically significant reduced alertness, is 20.5. No sex differences were noted and scores on the THAT did not differ with age, with no significant differences in the THAT score between those below and above 40 years of age.

\section{Relationship to sleepiness, fatigue, depression, and anxiety: sleep clinic patients}

Of the 264 patient charts included in this study, $54 \%(n=142)$ of the patients were women and $46 \%(n=122)$ were men, and the average age of the group was $45.3 \pm 14.2$ years. The results from the questionnaires and the average daytime sleep onset latency from the four MSLT sessions are shown in Table 1.

The group was divided into those with normal and impaired alertness based on the cutoff of 20.5 for the THAT (Table 2). A significant difference between those with normal and impaired alertness was found for all variables except for the ESS and the MSLT sleep onset latency. Effect size calculation (using Cohen's $d$ ) (Table 2) indicates large differences in fatigue (FSS) and depressive symptoms (CES-D scale) and moderate differences in anxiety (SAS) between those with normal and those with impaired alertness.

Correlational analyses (Table 3 ) revealed that alertness (as measured using the THAT scale) was modestly, significantly, and

Table I Questionnaire and MSLT results

\begin{tabular}{lll}
\hline Measure & Average $( \pm$ SD) & \% above cutoff \\
\hline THAT - alertness & $24.5 \pm 9.2$ & 36.7 (THAT $\leq 20.5)$ \\
ESS - EDS & $9.4 \pm 5.7$ & 41.1 (ESS $>10)$ \\
MSLT - EDS & $10.7 \pm 5.3$ & $73.7(\mathrm{SOL} \leq 10 \mathrm{~min})$ \\
FSS - fatigue & $5.0 \pm 1.3$ & $83.5(\mathrm{FSS} \geq 3.7)$ \\
CES-D - depressive symptoms & $21.6 \pm 13.4$ & $59.9(\mathrm{CES}-\mathrm{D} \geq 16)$ \\
SAS - anxiety symptoms & $35.9 \pm 13.0$ & $24.0(\mathrm{SAS} \geq 45)$ \\
\hline
\end{tabular}

Abbreviations: THAT, Toronto Hospital Alertness Test; ESS, Epworth Sleepiness Scale; MSLT, Multiple Sleep Latency Test; SOL, sleep onset latency; FSS, Fatigue Severity Scale; CES-D, Centre for Epidemiological Studies in Depression; SAS, Zung Self-Rating Anxiety Scale; EDS, excessive daytime sleepiness.
Table 2 Normal and impaired alertness across study variables

\begin{tabular}{lllll}
\hline Measure & $\begin{array}{l}\text { Normal } \\
\text { alertness }\end{array}$ & $\begin{array}{l}\text { Impaired } \\
\text { alertness }\end{array}$ & $\begin{array}{l}\text { Significance } \\
(P \text {-value })\end{array}$ & $\begin{array}{l}\text { Effect size } \\
\text { (Cohen's } d)\end{array}$ \\
& $\begin{array}{l}\text { Average } \\
\text { Average }\end{array}$ & $\begin{array}{l}\text { Aver } \\
( \pm \text { SD })\end{array}$ & & \\
\hline ESS & $9.3 \pm 5.4$ & $9.6 \pm 6.2$ & 0.7 & -0.052 \\
MSLT & $10.2 \pm 5.5$ & $11.4 \pm 4.9$ & 0.1 & -0.230 \\
FSS & $4.6 \pm 1.3$ & $5.7 \pm 1.0$ & $<0.001$ & -0.948 \\
CES-D & $16.4 \pm 10.9$ & $30.6 \pm 12.5$ & $<0.001$ & -1.211 \\
SAS & $32.5 \pm 12.3$ & $41.9 \pm 12.0$ & $<0.001$ & -0.774 \\
\hline
\end{tabular}

Abbreviations: ESS, Epworth Sleepiness Scale; MSLT, Multiple Sleep Latency Test; FSS, Fatigue Severity Scale; CES-D, Centre for Epidemiological Studies in Depression; SAS, Zung Self-Rating Anxiety Scale.

negatively correlated with fatigue levels (FSS), depressive symptoms (CES-D), and anxiety symptoms (SAS). A strong, significant, and positive correlation was also noted between scores on the CES-D and the SAS, and the FSS was modestly but positively and significantly correlated with scores on the CES-D and the SAS.

To further explore the relationship among alertness, fatigue, sleepiness, depressive symptoms, and anxiety complaints, regression analyses were performed. A significant model was evident $\left(F=19.9, P<0.001\right.$, adjusted $\left.R^{2}=0.35\right)$ with scores on the CES-D $(P<0.001)$ and FSS $(P=0.006)$ scales emerging as significant predictors of scores on the THAT scale. Neither ESS $(P=0.98)$ nor FSS $(P=0.29)$ were found to be significant predictor variables. The model incorporating the ESS, FSS, CES-D, and SAS was found to explain half $\left(50 \%\right.$, adjusted $\left.R^{2}=0.50\right)$ of the variability in scores on the THAT. Conversely, a model incorporating age, sex, Apnea-Hypopnea Index, and MSLT sleep latency was found

Table 3 Correlational analyses

\begin{tabular}{llllll}
\hline Measure & CES-D & SAS & ESS & FSS & MSLT \\
\hline THAT & -0.5 & -0.4 & -0.37 & -0.4 & 0.03 \\
& $P<0.00 \mathrm{I}$ & $P<0.00 \mathrm{I}$ & $P=0.7$ & $P<0.00 \mathrm{I}$ & $P=0.8$ \\
CES-D & - & $0.6 \mathrm{I}$ & 0.06 & 0.3 & -0.07 \\
& & $P<0.00 \mathrm{I}$ & $P=0.6$ & $P=0.005$ & $P=0.6$ \\
SAS & $0.6 \mathrm{I}$ & - & 0.03 & 0.3 & -0.03 \\
& $P<0.00 \mathrm{I}$ & & $P=0.7$ & $P=0.02$ & $P=0.8$ \\
ESS & 0.06 & 0.03 & - & 0.2 & -0.01 \\
& $P=0.6$ & $P=0.7$ & & $P=0.09$ & $P=0.1$ \\
FSS & 0.3 & 0.3 & 0.2 & - & -0.05 \\
& $P=0.005$ & $P=0.02$ & $P=0.09$ & & $P=0.7$ \\
MSLT & -0.07 & -0.03 & -0.01 & -0.05 & - \\
& $P=0.6$ & $P=0.8$ & $P=0.1$ & $P=0.7$ & \\
AHI & 0.2 & 0.2 & 0.1 & 0.1 & -0.03 \\
& $P=0.03$ & $P=0.03$ & $P=0.2$ & $P=0.2$ & $P=0.8$ \\
\hline
\end{tabular}

Abbreviations: THAT, Toronto Hospital Alertness Test; ESS, Epworth Sleepiness Scale; MSLT, Multiple Sleep Latency Test; FSS, Fatigue Severity Scale; CES-D, Centre for Epidemiological Studies in Depression; SAS, Zung Self-Rating Anxiety Scale; AHI, Apnea-Hypopnea Index. 
to explain only $16 \%$ (adjusted $R^{2}=0.16$ ) of the variability in scores on the THAT.

\section{Discussion}

Daytime sleepiness and poor alertness are used interchangeably in the literature. ${ }^{23}$ However, the findings of this study support our previous hypothesis that daytime sleepiness is not the same as poor alertness. Daytime sleepiness, whether measured subjectively with the ESS or physiologically from the sleep onset latency obtained using the MSLT, did not differ among those with and without impaired alertness and did not predict scores on the THAT, thereby confirming that alertness is a separate construct from and not merely the opposite of sleepiness.

This study is the first to quantitatively define normal levels of alertness. Alertness is important for appropriate on-task performance and for the prevention of accidents. Unfortunately, there is as yet agreed-upon definition of alertness and no gold standard for the measurement of alertness. As such, the definition of the construct of alertness is still controversial and further research in this area is needed to clarify exactly how alertness differs from sleepiness. The purpose of this paper has been to show what steps we have taken to further characterize the concept of alertness as an entity distinct from sleepiness.

There is strong evidence that performance is not solely dependent on the degree of sleepiness. Pejovic et al ${ }^{24}$ investigated the impact of recovery sleep of 10 hours per night for three nights following six nights of mild sleep restriction (6 hours/night) in healthy young adults. Subjects did not exhibit any objective or subjective sleepiness after the recovery sleep, but their performance on a psychomotor vigilance task remained significantly impaired. This study demonstrates that with sleep restriction, recovery of neurocognitive deficits such as impaired performance may be slower when compared to improvements in excessive sleepiness. The continued performance decrements in the absence of sleepiness emphasizes the lack of association between sleepiness and vigilance and points to the strong influence of factors other than daytime sleepiness on performance. Alertness is potentially a good candidate factor, but further study will be needed to validate this hypothesis. Certainly, the aforementioned study emphasizes the need to look beyond sleepiness as the primary determinant of performance and an exploration of other factors such as alertness to characterize how sleep can impact performance is warranted.

In contrast to sleepiness, symptoms of depression and fatigue among our study subjects were found to have a strong and significant impact on levels of alertness. An association between fatigue and symptoms consistent with depression was noted. This may be expected as fatigue is a major symptom of depressive disorders. ${ }^{25}$ However, there is as yet no information on poor alertness as a symptom of depressive disorders.

Depressive disorders are characterized by impairments in both vigilance ${ }^{26}$ and attention. ${ }^{27}$ A study from our group reported that patients with untreated major depressive disorder performed poorly on the driving simulator. ${ }^{28}$ Greater workplace injuries and accidents have also been noted among firefighters with more depressive symptoms. ${ }^{29}$ The above could be explained by a reduction in alertness in those with depressive disorder as alertness has been linked to reduced visuospacial attention. ${ }^{30}$ Along these lines, our study ${ }^{31}$ has shown that treatment with mirtazapine improves driving performance despite the increased short-term sedation associated with mirtazapine.

\section{Conclusion}

The current study underscores that sleepiness and alertness are separate phenomena and not opposite poles of the same continuum. One limitation of this study is the use of only questionnaire data to explore alertness in sleep clinic patients. A further limitation is the use of only 60 control subjects to determine normative values of the THAT. It is notable that despite this low number of subjects, the cutoff of 20.5 for the THAT (calculated as twice the SD subtracted from the mean) obtained in our study is identical to the cutoff of 20.5 for the THAT calculated based on a larger study of 1,010 normal subjects. ${ }^{4}$ With an established normative values for alertness, further studies can utilize these to investigate linkages between impaired alertness and changes in sleep architectural variables in patients with sleep disorders, particularly in insomnia patients where fatigue, but not daytime sleepiness, predominates. Additionally, given that symptoms indicative of depression are predictive of levels of alertness, future studies are also needed to explore the contribution of impaired alertness to neurovegetative symptoms in individuals with depressed mood.

\section{Acknowledgment}

The abstract of this paper was presented at the 27th Annual Meeting of the Associated Professional Sleep Societies, Baltimore, MD; 2013. The abstract was published in Journal of Sleep and Sleep Disorders Research, vol 36, 2013.

\section{Disclosure}

The authors report no conflicts of interest in this work. 


\section{References}

1. Shapiro CM, Auch C, Reimer M, et al. A new approach to the construct of alertness. J Psychosom Res. 2006;60(6):595-603.

2. Ohayon MM, Dauvilliers Y, Reynolds CF 3rd. Operational definitions and algorithms for excessive sleepiness in the general population: implications for DSM-5 nosology. Arch Gen Psychiat. 2012;69:71-79.

3. Ionescu D, Driver HS, Heon E, Flanagan J, Shapiro CM. Sleep and daytime sleepiness in retinitis pigmentosa patients. J Sleep Res. 2001;10(4):329-335.

4. Dean B, Aguilar D, Shapiro CM, et al. Impaired health status, daily functioning, and work productivity in adults with excessive sleepiness. J Occup Environ Med. 2010;52(2):144-149.

5. Bonnet MH, Arand DL. Impact of motivation on Multiple Sleep Latency Test and Maintenance of Wakefulness Test measurements. J Clin Sleep Med. 2005;1(4):386-390.

6. Littner MR, Kushida C, Wise M, et al; Standards of Practice Committee of the American Academy of Sleep Medicine. Practice parameters for clinical use of the Multiple Sleep Latency Test and the maintenance of wakefulness test. Sleep. 2005;28(1):113-121.

7. Radloff L. The CES-D Scale: a self-report depression scale for research in the general population. App Psychol Meas. 1977;385-401.

8. Valentine AD, Meyers CA. Cognitive and mood disturbance as causes and symptoms of fatigue in cancer patients. Cancer. 2001;92: 1694-1698.

9. Bardwell WA, Moore P, Ancoli-Israel S, Dimsdale JE. Fatigue in obstructive sleep apnea: driven by depressive symptoms instead of apnea severity? Am J Psychiat. 2003;160:350-355.

10. Hossain JL, Ahmad P, Reinish LW, Kayumov L, Hossain NK, Shapiro CM. Subjective fatigue and subjective sleepiness: two independent consequences of sleep disorders? J Sleep Res. 2005; 14(3):245-253.

11. Ickmans K, Meeus M, Kos D, et al. Cognitive performance is of clinical importance, but is unrelated to pain severity in women with chronic fatigue syndrome. Clin Rheumatol. 2013;32(10):1475-1485.

12. Sinclair KL, Ponsford JL, Rajaratnam SM, Anderson C. Sustained attention following traumatic brain injury: use of the Psychomotor Vigilance Task. J Clin Exp Neuropsychol. 2013;35(2):210-224.

13. McDonald E, Cope H, David A. Cognitive impairment in patients with chronic fatigue: a preliminary study. J Neurol Neurosurg Psychiat. 1993;56(7):812-815.

14. Short K, McCabe M, Tooley G. Cognitive functioning in chronic fatigue syndrome and the role of depression, anxiety, and fatigue. J Psychosom Res. 2002;2(6):475-483.

15. Joyce E, Blumenthal S, Wessely S. Memory, attention, and executive function in chronic fatigue syndrome. J Neurol Neurosurg Psychiatry. 1996;60(5):495-503.
16. Capuron L, Welberg L, Heim C, et al. Cognitive dysfunction relates to subjective report of mental fatigue in patients with chronic fatigue syndrome. Neuropsychopharmacology. 2006;31(8):1777-1784.

17. Zung WWK. A rating instrument for anxiety disorders. Psychosomatics. $1971 ; 12: 371-379$.

18. Johns MW. A new method for measuring daytime sleepiness: the Epworth Sleepiness Scale. Sleep. 1991;14(6):540-545.

19. Krupp LB, LaRocca NG, Muir-Nash J, Steinberg AD. The Fatigue Severity Scale. Application to patients with multiple sclerosis and systemic lupus erythematosus. Arch Neurol. 1989;46:1121-1123.

20. Richardson GS, Carskadon MA, Flagg W, Van den Hoed J, Dement WC, Mitler MM. Excessive daytime sleepiness in man: multiple sleep latency measurement in narcoleptic and control subjects. Electroencephalogr Clin Neurophysiol. 1978;45(5):621-627.

21. Rosenthal LD, Dolan DC. The Epworth Sleepiness Scale in the identification of obstructive sleep apnea. J Nerv Ment Dis. 2008; 196(5):429-431.

22. Cohen, J. Statistical power analysis for the behavioral sciences. 2nd ed. Hillsdale, NJ: Lawrence Earlbaum Associates; 1998.

23. Aston-Jones G. Brain structures and receptors involved in alertness. Sleep Med. 2005;6(Suppl 1):S3-S7.

24. Pejovic S, Basta M, Vgontzas AN, et al. Effects of recovery sleep after one work week of mild sleep restriction on interleukin-6 and cortisol secretion and daytime sleepiness and performance. Am J Physiol Endocrinol Metab. 2013;305(7):E890-E896.

25. American Psychiatric Association. Diagnostic and statistical manual of mental disorders. 4th ed. Washington, DC; 2000.

26. Kertzman S, Reznik I, Hornik-Lurie T, Weizman A, Kotler M, Amital D. Stroop performance in major depression: selective attention impairment or psychomotor slowness? J Affect Disord. 2010;122(1-2):167-173.

27. Godard J, Grondin S, Baruch P, Lafleur MF. Psychosocial and neurocognitive profiles in depressed patients with major depressive disorder and bipolar disorder. Psychiatry Res. 2011;190(2-3):244-252.

28. Bulmash EL, Moller HJ, Kayumov L, Shen J, Wang X, Shapiro CM. Psychomotor disturbance in depression: assessment using a driving simulator paradigm. J Affect Disord. 2006;93(1-3):213-218.

29. Chung YK, Park CY. The effects of injury and accidents on self-rated depression in male municipal firefighters. Saf Health Work. 2011; 2(2):158-168.

30. Schock L, Schwenzer M, Sturm W, Mathiak K. Alertness and visuospatial attention in clinical depression. BMC Psychiat. 2011;11:78.

31. Shen J, Moller HJ, Wang X, et al. Mirtazapine, a sedating antidepressant, and improved driving safety in patients with major depressive disorder: a prospective, randomized trial of 28 patients. J Clin Psychiatry. 2009; 70(3):370-377.
Nature and Science of Sleep

\section{Publish your work in this journal}

Nature and Science of Sleep is an international, peer-reviewed, open access journal covering all aspects of sleep science and sleep medicine, including the neurophysiology and functions of sleep, the genetics of sleep, sleep and society, biological rhythms, dreaming, sleep disorders and therapy, and strategies to optimize healthy sleep. The journal welcomes

\section{Dovepress}

original research, clinical \& epidemiological studies, reviews \& evaluations, case reports and extended reports. The manuscript management system is completely online and includes a very quick and fair peerreview system, which is all easy to use. Visit http://www.dovepress.com/ testimonials.php to read real quotes from published authors. 